\section{Leukocyte counts are associated with components of the metabolic syndrome}

There is a positive association between leukocyte counts and vascular complications of type 2 diabetes mellitus (T2DM), such as carotid atherosclerosis, cardiovascular disease, and mortality from coronary heart disease and cerebrovascular disease. It is thought that leukocytes might also contribute to the pathogenesis of the metabolic syndrome and atherosclerosis. Researchers in Taiwan have investigated the association of white blood cell counts with presence of the metabolic syndrome and ischemic macrovascular diseases (including coronary artery and ischemic cerebrovascular diseases) in patients with T2DM.

The study enrolled 1,872 patients with T2DM who entered a disease management program between January 2002 and December 2004. In total, 1,367 patients $(73 \%)$ met the criteria for the metabolic syndrome, $122(7 \%)$ had coronary artery disease and 111 (6\%) had ischemic cerebrovascular disease. Patients were divided into quartiles according to total white blood cell, neutrophil, monocyte, and lymphocyte counts. The prevalence of the metabolic syndrome alone and particular components of the metabolic syndrome (central obesity, hypertension, hypertriglyceridemia and low HDL cholesterol levels) were significantly associated with peripheral total and differential leukocyte counts $\left(P_{\text {trend }}<0.05\right)$. Ischemic macrovascular diseases were also associated with increasing quartiles of total and differential leukocyte count (all $P<0.01$ ).

The authors conclude that peripheral total and differential leukocyte counts seem to be associated with a clustering of metabolic syndrome components and ischemic macrovascular diseases in patients with T2DM.

Original article Tsai JC-R et al. (2006) Association of peripheral total and differential leukocyte counts with metabolic syndrome and risk of ischemic cardiovascular diseases in patients with type 2 diabetes mellitus. Diabetes Metab Res Rev [doi: 10.1002/dmrr.647]

\section{Pacemaker diagnostics in premature atrial contraction suppression}

Patients requiring a pacemaker often have atrial fibrillation (AF), which increases the risk of thromboembolism and, consequently, the risk of morbidity and mortality. To maintain sinus rhythms, atrial pacing algorithms have been incorporated into pacemaker software. These algorithms target modification of atrial substrate and suppress AF triggers, including premature atrial contractions (PACs), believed to be a primary inducer of AF. Using data from dual-chamber pacemakers with advanced diagnostic functions, Yang et al. have identified various AF onset patterns in patients with paroxysmal $A F$ and described those patients who might benefit most from PAC-suppressing pacing algorithms.

Patients with high PAC activity in the $5 \mathrm{~min}$ before AF onset had fewer recurrent AF episodes than those with moderate PAC activity, and a lower mean episode duration than those with low PAC activity, resulting in reduced AF burden. These patients with high PAC activity most likely have little alteration in atrial substrate, and their AF development is probably induced by high trigger activity. These patients (who the authors believe to be those at an early disease stage) are most likely to respond to PAC-suppressing pacing algorithms.

Patients with low PAC activity before AF onset, on the other hand, have a prolonged arrhythmia episode duration and high AF burden, probably caused by a high atrial substrate factor. In these patients, severe structural and electrophysiological changes in the atrial myocardium, induced by a few PACs or even occurring in the absence of PACs, are presumed to be key in initiating AF. Atrial substrate factor serves to sustain rather than trigger arrhythmia, and patients with low PAC activity are less likely to respond to the pacing algorithms.

Original article Yang A et al. (2006) Identification of "substrate fibrillators" and "trigger fibrillators" by pacemaker diagnostics. Heart Rhythm 3: 682-688

\section{Manual versus automated chest compression for cardiopulmonary resuscitation}

Quality of cardiopulmonary resuscitation (CPR) is likely to be one of the key determinants of successful resuscitation following an out-ofhospital cardiac arrest. Consistent compressions can be difficult to maintain, however, and the quality of both in-hospital and out-ofhospital CPR is sometimes poor. Mechanical devices have been developed with the aim 